\title{
Estimation of stature by using the dimensions of the right hand and right foot in Han Chinese adults
}

\author{
Xinghua Zhang ${ }^{1 \dagger}$, Yu Wei ${ }^{1 \dagger}$, Lianbin Zheng ${ }^{1{ }^{\dagger *}}$, Keli $\mathrm{Yu}^{1 \dagger}$, Dapeng Zhao ${ }^{1}$, Jinping $\mathrm{Bao}^{2}$, \\ Yonglan $\mathrm{Li}^{3}$, Shunhua $\mathrm{Lu}^{3}$, Huanjiu $\mathrm{Xi}^{4}$, Guochang $\mathrm{Xu}^{5} \&$ Youfeng $\mathrm{Wen}^{4}$ \\ ${ }^{1}$ Tianjin Key Laboratory of Animal and Plant Resistance, College of Life Sciences, Tianjin Normal University, Tianjin 300387, China; \\ ${ }^{2}$ Institute of Sports Science, Tianjin Normal University, Tianjin 300387, China; \\ ${ }^{3}$ College of Life Science and Technology, Inner Mongolia Normal University, Hohhot 010022, China; \\ ${ }^{4}$ Institute of Biological Anthropology in Liaoning Medical University, Jinzhou 121000, China; \\ ${ }^{5}$ Institute of Anthropology, Nanyang Institute of Technology, Nanyang 473004, China
}

Received June 3,2016; accepted June 20, 2016; published online December 21, 2016

\begin{abstract}
The Han Chinese people are the main ethnic group in China and the largest ethnic group in the world. The dimensions of the hands and feet have been successfully used for the estimation of stature. A total of 26,927 healthy adult subjects, comprising 13,221 men and 13,706 women, were recruited. The survey samples were chosen through random cluster sampling. The mean values were significantly higher in men than those in women for all measurements $(P<0.001)$. All the measurements showed a statistically significant correlation with stature $(P<0.01)$. The combination of the right hand length and the right foot length was the best predictor of stature because it had the lowest standard error of estimate. The use of multiple regression equations yielded better results than did the use of linear regression equations. The accuracy of stature prediction ranged from \pm 4.81 to $\pm 6.39 \mathrm{~cm}$. The present study was of great importance with regards to improving the physical anthropology database of ethnic groups in China.
\end{abstract}

stature, forensic anthropology, anthropometry, foot length, foot width, Han Chinese

Citation: Zhang, X., Wei, Y., Zheng, L., Yu, K., Zhao, D., Bao, J., Li, Y., Lu, S., Xi, H., Xu, G., and Wen, Y. (2017). Estimation of stature by using the dimensions of the right hand and right foot in Han Chinese adults. Sci China Life Sci 60, 81-90. doi: 10.1007/s11427-016-0051-8

\section{INTRODUCTION}

Forensic scientists are interested in the estimation of stature because of its importance in personal identification. There is a relationship between each part of the body and the whole body. The hand and foot dimensions may represent a person's identity. Human stature is an anatomical complex of linear dimensions (Moorthy et al., 2014; Uhrová et al., 2015; Zaher et al., 2011).

Many researchers have found a relationship between stature

$\dagger$ Contributed equally to this work

*Corresponding author (email: zhenglianbin@sina.com) and several different body parts such as the hands and feet (Abdel-Malek et al., 1990; Agnihotri et al., 2007; Agnihotri et al., 2008; Atamturk and Duyar, 2008; Cervantes et al., 1988; Fessler et al., 2005; Gordon and Buikstra, 1992; Habib and Kamal, 2010; Ishak et al., 2012; Kanchan et al., 2008; Kanchan et al., 2010; Krishan, 2008a; Krishan, 2008b; Ozden et al., 2005; Rastogi et al., 2008; Reel et al., 2012; Sanli et al., 2005; Saxena, 1984; Sen and Ghosh, 2008; Zeybek et al., 2008), face and head (Baume and Buschang, 1983; Hautvast, 1971; Sahni et al., 2010), vertebral column (Jason and Taylor, 1995; Karakas et al., 2011; Nagesh and Pradeep Kumar, 2006; Pelin et al., 2005; Pininski and Brits, 2014; Qing et al., 2013; Terazawa et al., 1990; Tibbetts, 1981; Torimitsu 
et al., 2014; Zhang et al., 2008; Zhang et al., 2015), and upper-limb and lower-limb bones (Ahmed, 2013; Aldegheri and Dall'Oca, 2001; Choi et al., 1997; Ikeda et al., 1977; Lavelle, 1977; Mahakkanukrauh et al., 2011; Stanitski, 2004; Trotter and Gleser, 1958). The results have supported their claim that stature can be estimated successfully from all kinds of body parts. The hand and foot dimensions have been successfully used for the estimation of stature. The standards for the estimation of stature are based on anatomical and mathematical methods.

A previous study has confirmed that the correlation coefficients for stature and right hand/foot length are equal to those for stature and left hand/foot length (Uhrová et al., 2015). The aim of this study was to assess the relationship of the dimensions of the right hand and right foot with the stature, and to provide equations for stature estimation in Han Chinese adults. The Han Chinese people are the main ethnic group in China and the largest ethnic group in the world. The results of our research have practical use in the forensic field as well as in the field of sport and physical anthropology. The present study is of great significance in improving the physical anthropology database of ethnic groups in China.

The fact that the equations derived from one population cannot be used for other populations needs to be taken into the consideration, as body dimensions show ethnic variations due to genetic factors and environmental conditions. A single formula cannot represent the nationwide or worldwide population (Fawzy and Kamal, 2010; Jasuja et al., 1991; Krishan et al., 2012). It would be useful, however, to assess whether this method of stature estimation has forensic potential in other populations.

\section{RESULTS}

The descriptive statistical results of means and standard deviations for stature, right hand measurements, and right foot measurements are shown in Table 1. The mean stature in men was higher than that in women. The mean values were significantly higher in men than those in women for all measurements (Table 1).

The correlation coefficients for stature and right hand/foot measurements are shown in Table 2. All the measurements showed a statistically significant correlation with stature $(P<0.01)$. In men, the highest correlation coefficient was between the stature and right foot length $(r=0.602)$, and the lowest was between the stature and right hand breadth $(r=0.240)$. Similarly, in women, the right foot length resulted in the highest correlation coefficient $(r=0.565)$, whereas the right hand breadth showed the lowest correlation $(r=0.175)$ with stature.

The linear regression equations for the estimation of stature from the right hand and right foot dimensions are shown in Table 3. The regression coefficients are significant for all parameters. For the analysis of right hand and right foot measurements, the standard error of estimate (SEE) ranged between \pm 5.251 and $\pm 6.385 \mathrm{~cm}$ for men and between \pm 4.850 and $\pm 5.788 \mathrm{~cm}$ for women. In Table 3 , the right foot lengths showed the lowest SEE. Therefore, the right foot length would provide the most reliable stature estimation.

Tables 4 and 5 show that the combination of right hand/foot length is the best predictor of stature because it had the lowest SEE; for men, it was the combination of right hand length and right foot length $(\mathrm{SEE} \pm 5.188 \mathrm{~cm})$, and for women it was the combination of right foot length and right hand length $(\mathrm{SEE} \pm 4.822 \mathrm{~cm})$. Tables 4 and 5 show that multiple regression equations resulted in lower SEE values than did linear regression equations, ranging between \pm 5.179 and $\pm 6.128 \mathrm{~cm}$ for men, and between \pm 4.812 and $\pm 5.636 \mathrm{~cm}$ for women.

\section{DISCUSSION}

In this study of Han Chinese adults, the stature and right hand/foot dimensions were found to be higher in men than in women. The results were consistent with those of previous findings (Agnihotri et al., 2008; Ahmed, 2013; Ishak et al., 2012; Kanchan et al., 2008; Kanchan et al., 2010; Krishan and Sharma, 2007; Rastogi et al., 2008; Sen and Ghosh, 2008; Uhrová et al., 2013; Uhrová et al., 2015; Zeybek et al., 2008; Zheng et al., 2013; Zheng et al., 2015). The values for Han Chinese men were higher than those for Han Chinese women, with a significant difference between sexes (Table 1). The mean values of men were higher than those of women owing to their later maturity and growth cessation. Many previous studies have confirmed the presence of a positive correlation between several different body parts and stature (Agnihotri et al., 2007; Ahmed, 2013; Ishak et al., 2012; Krishan and Sharma, 2007; Uhrová et al., 2015). We found that the highest correlation coefficient was between stature and right foot length in men and women, whereas the correlation coefficient between stature and right hand breadth was the lowest.

The study showed that the ratios of foot dimensions to stature changed considerably with age and sex. Consequently, the regression equations that include these variables yielded more reliable results (Atamturk and Duyar, 2008). Our results indicated that age and sex should be taken into consideration when predicting human stature.

The results of this study showed that the right foot lengths with the lowest SEE would provide the most reliable stature estimation. The regression equations of stature from the right foot length for Han Chinese men and women were both statistically significant in the test of the regression effect. There was a significant linear regression relationship between stature and right foot length, and stature could be estimated by using the following regression equations: $S=88.522+3.225 \mathrm{RFL}$ for men and $S=89.811+2.941 \mathrm{RFL}$ for women. The SEE values of multiple regression equations 
Table 1 Descriptive statistics: stature, right hand dimensions $(\mathrm{cm})$, and right foot dimensions $(\mathrm{cm})$ in men and women ${ }^{\text {a) }}$

\begin{tabular}{|c|c|c|c|c|c|c|c|c|}
\hline \multirow{2}{*}{ Ethnic groups } & \multirow{2}{*}{ Age groups (Years) } & \multirow{2}{*}{ Value of $r$} & \multicolumn{2}{|c|}{ Men } & \multicolumn{2}{|c|}{ Women } & \multicolumn{2}{|c|}{$t$-test } \\
\hline & & & Mean & $\mathrm{SD}$ & Mean & $\mathrm{SD}$ & $t$-value & $P$ \\
\hline \multirow{30}{*}{ Northern Han } & $19-29$ & $\mathrm{~S}$ & 171.280 & 6.034 & 158.667 & 5.459 & 52.006 & 0.000 \\
\hline & Men $(n=1,131)$ & RHL & 18.199 & 1.972 & 16.923 & 1.627 & 16.749 & 0.000 \\
\hline & Women $(n=1,121)$ & RHB & 8.101 & 0.569 & 7.193 & 0.540 & 38.854 & 0.000 \\
\hline & & RFL & 24.511 & 1.265 & 22.372 & 1.174 & 41.573 & 0.000 \\
\hline & & RFB & 9.404 & 0.705 & 8.491 & 0.646 & 32.034 & 0.000 \\
\hline & $30-39$ & $\mathrm{~S}$ & 168.343 & 5.665 & 157.278 & 5.517 & 47.886 & 0.000 \\
\hline & Men $(n=1,149)$ & RHL & 18.232 & 1.813 & 17.046 & 1.736 & 16.174 & 0.000 \\
\hline & Women $(n=1,193)$ & RHB & 8.255 & 0.525 & 7.431 & 0.521 & 38.114 & 0.000 \\
\hline & & RFL & 24.266 & 1.205 & 22.413 & 1.151 & 38.049 & 0.000 \\
\hline & & RFB & 9.515 & 0.727 & 8.719 & 0.617 & 28.612 & 0.000 \\
\hline & $40-49$ & $\mathrm{~S}$ & 167.376 & 5.955 & 156.712 & 5.780 & 44.850 & 0.000 \\
\hline & Men $(n=1,212)$ & RHL & 18.339 & 1.827 & 17.166 & 1.560 & 17.040 & 0.000 \\
\hline & Women $(n=1,224)$ & RHB & 8.330 & 0.551 & 7.491 & 0.518 & 38.753 & 0.000 \\
\hline & & RFL & 24.439 & 1.238 & 22.488 & 1.174 & 39.912 & 0.000 \\
\hline & & RFB & 9.622 & 0.681 & 8.764 & 0.628 & 32.348 & 0.000 \\
\hline & $50-59$ & $\mathrm{~S}$ & 166.584 & 6.002 & 155.266 & 5.509 & 47.806 & 0.000 \\
\hline & Men $(n=1,170)$ & RHL & 18.397 & 1.730 & 17.225 & 1.617 & 17.032 & 0.000 \\
\hline & Women $(n=1,196)$ & RHB & 8.287 & 0.578 & 7.517 & 0.529 & 33.811 & 0.000 \\
\hline & & RFL & 24.376 & 1.262 & 22.443 & 1.226 & 37.803 & 0.000 \\
\hline & & RFB & 9.624 & 0.695 & 8.776 & 0.643 & 30.789 & 0.000 \\
\hline & $60+$ & $\mathrm{S}$ & 165.139 & 5.907 & 152.943 & 5.431 & 51.945 & 0.000 \\
\hline & Men $(n=1,180)$ & RHL & 18.393 & 1.810 & 17.304 & 1.488 & 15.873 & 0.000 \\
\hline & Women $(n=1,158)$ & RHB & 8.254 & 0.578 & 7.536 & 0.485 & 32.518 & 0.000 \\
\hline & & RFL & 24.310 & 1.207 & 22.271 & 1.111 & 42.481 & 0.000 \\
\hline & & RFB & 9.595 & 0.660 & 8.726 & 0.681 & 31.317 & 0.000 \\
\hline & Northern Han total & $\mathrm{S}$ & 167.712 & 6.254 & 156.164 & 5.867 & 103.161 & 0.000 \\
\hline & Men $(n=5,842)$ & RHL & 18.313 & 1.832 & 17.134 & 1.613 & 37.002 & 0.000 \\
\hline & Women $(n=5,892)$ & RHB & 8.247 & 0.566 & 7.436 & 0.533 & 79.922 & 0.000 \\
\hline & & RFL & 24.380 & 1.238 & 22.399 & 1.170 & 89.090 & 0.000 \\
\hline & & RFB & 9.554 & 0.698 & 8.698 & 0.651 & 68.662 & 0.000 \\
\hline \multirow{30}{*}{ Southern Han } & $19-29$ & $\mathrm{~S}$ & 169.554 & 5.956 & 157.300 & 5.198 & 61.413 & 0.000 \\
\hline & Men $(n=1,595)$ & RHL & 18.042 & 1.464 & 16.744 & 1.314 & 26.158 & 0.000 \\
\hline & Women $(n=1,551)$ & RHB & 8.005 & 0.404 & 7.085 & 0.376 & 66.136 & 0.000 \\
\hline & & RFL & 24.246 & 1.148 & 22.055 & 1.023 & 56.473 & 0.000 \\
\hline & & RFB & 9.299 & 0.587 & 8.421 & 0.538 & 43.723 & 0.000 \\
\hline & $30-39$ & $\mathrm{~S}$ & 166.521 & 6.121 & 155.723 & 5.167 & 51.671 & 0.000 \\
\hline & Men $(n=1,386)$ & RHL & 17.958 & 1.545 & 16.886 & 1.336 & 20.106 & 0.000 \\
\hline & Women $(n=1,535)$ & RHB & 8.162 & 0.427 & 7.353 & 0.383 & 53.993 & 0.000 \\
\hline & & RFL & 24.003 & 1.151 & 22.145 & 1.081 & 44.968 & 0.000 \\
\hline & & RFB & 9.379 & 0.628 & 8.668 & 0.569 & 32.107 & 0.000 \\
\hline & $40-49$ & $\mathrm{~S}$ & 165.079 & 6.262 & 155.158 & 5.373 & 47.530 & 0.000 \\
\hline & Men $(n=1,499)$ & RHL & 17.945 & 1.679 & 17.019 & 1.363 & 16.944 & 0.000 \\
\hline & Women $(n=1,613)$ & RHB & 8.231 & 0.454 & 7.453 & 0.396 & 51.039 & 0.000 \\
\hline & & RFL & 24.003 & 1.222 & 22.232 & 1.101 & 42.509 & 0.000 \\
\hline & & RFB & 9.492 & 0.642 & 8.748 & 0.577 & 34.011 & 0.000 \\
\hline & $50-59$ & $\mathrm{~S}$ & 164.124 & 6.276 & 153.494 & 5.438 & 50.000 & 0.000 \\
\hline & Men $(n=1,442)$ & RHL & 18.190 & 1.466 & 17.012 & 1.314 & 23.363 & 0.000 \\
\hline & Women $(n=1,595)$ & RHB & 8.224 & 0.430 & 7.478 & 0.396 & 49.716 & 0.000 \\
\hline & & RFL & 24.023 & 1.224 & 22.197 & 1.114 & 43.033 & 0.000 \\
\hline & & RFB & 9.532 & 0.646 & 8.746 & 0.590 & 35.026 & 0.000 \\
\hline & $60+$ & $\mathrm{S}$ & 162.023 & 6.176 & 151.106 & 5.832 & 49.603 & 0.000 \\
\hline & Men $(n=1,457)$ & RHL & 18.036 & 1.438 & 17.079 & 1.375 & 18.553 & 0.000 \\
\hline & Women $(n=1,520)$ & RHB & 8.108 & 0.429 & 7.442 & 0.381 & 44.831 & 0.000 \\
\hline & & RFL & 23.800 & 1.192 & 22.046 & 1.092 & 41.893 & 0.000 \\
\hline & & RFB & 9.511 & 0.670 & 8.704 & 0.610 & 34.370 & 0.000 \\
\hline & Southern Han total & $\mathrm{S}$ & 165.527 & 6.665 & 154.566 & 5.795 & 108.335 & 0.000 \\
\hline & Men $(n=7,379)$ & RHL & 18.034 & 1.523 & 16.948 & 1.345 & 46.638 & 0.000 \\
\hline & Women $(n=7,814)$ & RHB & 8.144 & 0.437 & 7.363 & 0.413 & 113.173 & 0.000 \\
\hline & & RFL & 24.019 & 1.196 & 22.136 & 1.086 & 101.708 & 0.000 \\
\hline & & RFB & 9.440 & 0.641 & 8.658 & 0.590 & 78.326 & 0.000 \\
\hline \multirow{5}{*}{ Han Chinese } & Han Chinese total & $\mathrm{S}$ & 166.492 & 6.576 & 155.253 & 5.879 & 147.960 & 0.000 \\
\hline & $\operatorname{Men}(n=13,221)$ & RHL & 18.158 & 1.672 & 17.028 & 1.469 & 58.917 & 0.000 \\
\hline & Women $(n=13,706)$ & RHB & 8.189 & 0.501 & 7.394 & 0.470 & 134.391 & 0.000 \\
\hline & & RFL & 24.179 & 1.228 & 22.249 & 1.130 & 134.239 & 0.000 \\
\hline & & RFB & 9.490 & 0.669 & 8.675 & 0.617 & 103.951 & 0.000 \\
\hline
\end{tabular}

a) $S$, stature; RHL, right hand length; RHB, right hand breadth; RFL, right foot length; RFB, right foot breadth; SD, standard deviation. 
Table 2 Correlation between stature and dimensions of the right hand and right foot ${ }^{\mathrm{a}}$

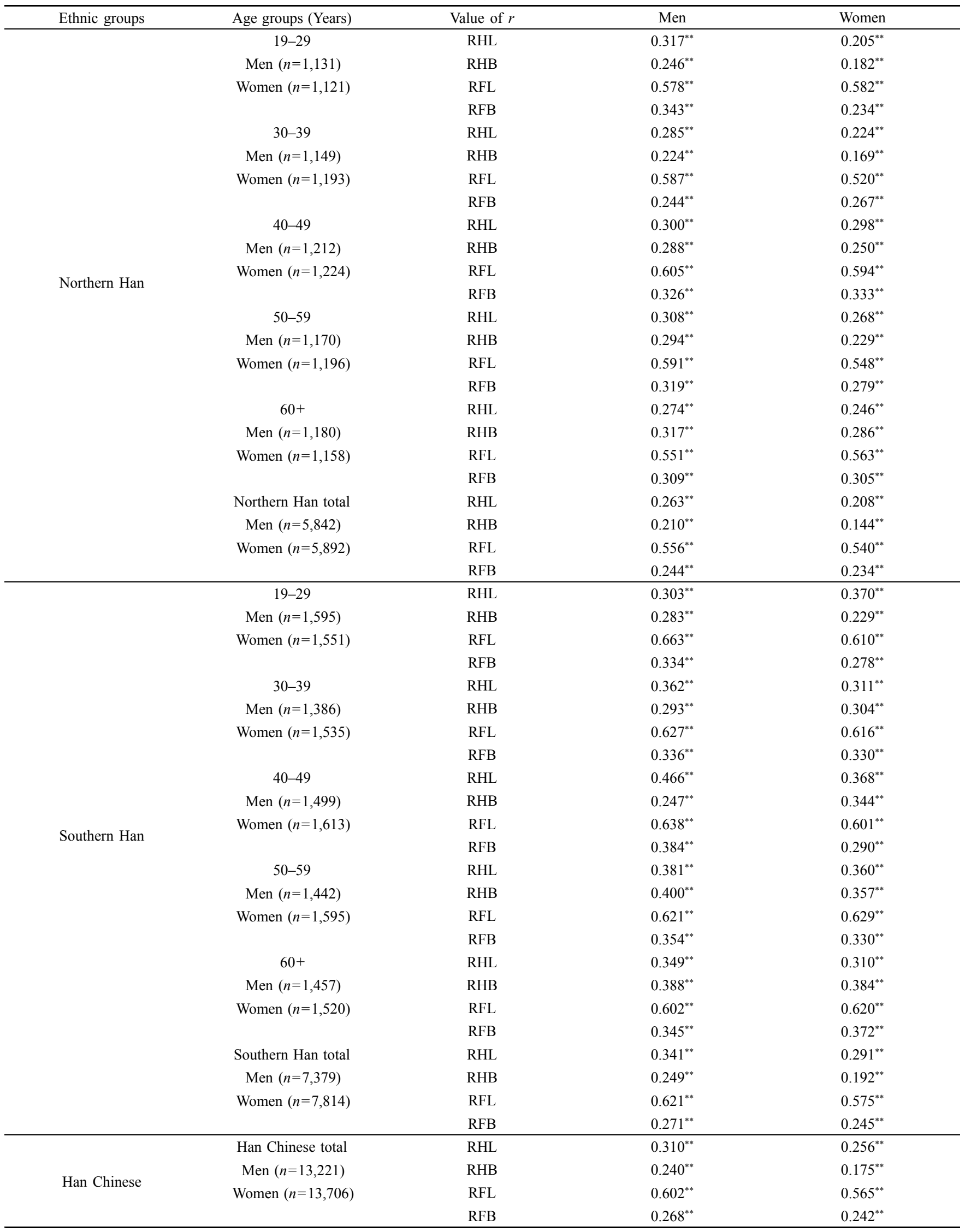

a) $r$, correlation coefficient. **, Significant at $\leqslant 0.01$. 
Table 3 Linear regression equations for stature estimation $(\mathrm{cm})$ from right hand $/$ foot dimensions ${ }^{\text {a) }}$

\begin{tabular}{|c|c|c|c|c|c|c|c|}
\hline \multirow{2}{*}{ Ethnic groups } & \multirow{2}{*}{ Age groups (Years) } & \multicolumn{3}{|c|}{ Men } & \multicolumn{3}{|c|}{ Women } \\
\hline & & Equation & $R^{2}$ & SEE & Equation & $R^{2}$ & SEE \\
\hline \multirow{24}{*}{ Northern Han } & $19-29$ & $S=153.657+0.968 \mathrm{RHL}$ & 0.100 & \pm 5.726 & $S=147.022+0.688 \mathrm{RHL}$ & 0.042 & \pm 5.345 \\
\hline & Men $(n=1,131)$ & $S=150.147+2.609 \mathrm{RHB}$ & 0.060 & \pm 5.851 & $S=145.445+1.838 \mathrm{RHB}$ & 0.033 & \pm 5.370 \\
\hline & Women $(n=1,121)$ & $S=103.689+2.758 \mathrm{RFL}$ & 0.334 & \pm 4.925 & $S=98.195+2.703 \mathrm{RFL}$ & 0.338 & \pm 4.443 \\
\hline & & $S=143.679+2.935 \mathrm{RFB}$ & 0.118 & \pm 5.670 & $S=141.897+1.975 \mathrm{RFB}$ & 0.055 & \pm 5.310 \\
\hline & $30-39$ & $S=152.120+0.890 \mathrm{RHL}$ & 0.081 & \pm 5.433 & $S=145.136+0.712 \mathrm{RHL}$ & 0.050 & \pm 5.379 \\
\hline & Men $(n=1,149)$ & $S=148.411+2.415 \mathrm{RHB}$ & 0.050 & \pm 5.524 & $S=143.983+1.789 \mathrm{RHB}$ & 0.029 & \pm 5.440 \\
\hline & Women $(n=1,193)$ & $\mathrm{S}=101.351+2.761 \mathrm{RFL}$ & 0.345 & \pm 4.587 & $S=101.452+2.491 \mathrm{RFL}$ & 0.270 & \pm 4.715 \\
\hline & & $S=150.253+1.901 \mathrm{RFB}$ & 0.060 & \pm 5.496 & $S=136.454+2.388 \mathrm{RFB}$ & 0.071 & \pm 5.319 \\
\hline & $40-49$ & $S=149.426+0.979 \mathrm{RHL}$ & 0.090 & \pm 5.682 & $S=137.776+1.103 \mathrm{RHL}$ & 0.089 & \pm 5.520 \\
\hline & $\operatorname{Men}(n=1,212)$ & $S=141.453+3.112 \mathrm{RHB}$ & 0.083 & \pm 5.705 & $S=135.836+2.787 \mathrm{RHB}$ & 0.062 & \pm 5.599 \\
\hline & Women $(n=1,224)$ & $S=96.222+2.911 \mathrm{RFL}$ & 0.367 & \pm 4.741 & $S=90.904+2.926 \mathrm{RFL}$ & 0.353 & \pm 4.650 \\
\hline & & $S=139.970+2.848 \mathrm{RFB}$ & 0.106 & \pm 5.633 & $S=129.851+3.065 \mathrm{RFB}$ & 0.111 & \pm 5.452 \\
\hline & $50-59$ & $S=145.536+1.123 \mathrm{RHL}$ & 0.095 & \pm 6.135 & $S=139.568+0.911 \mathrm{RHL}$ & 0.072 & \pm 5.310 \\
\hline & Men $(n=1,170)$ & $S=139.508+3.223 \mathrm{RHB}$ & 0.087 & \pm 6.162 & $S=137.368+2.381 \mathrm{RHB}$ & 0.052 & \pm 5.365 \\
\hline & Women $(n=1,196)$ & $S=94.392+2.948 \mathrm{RFL}$ & 0.349 & \pm 5.201 & $S=99.961+2.464 \mathrm{RFL}$ & 0.301 & \pm 4.609 \\
\hline & & $S=137.896+2.942 \mathrm{RFB}$ & 0.102 & \pm 6.111 & $S=134.264+2.393 \mathrm{RFB}$ & 0.078 & \pm 5.292 \\
\hline & $60+$ & $S=148.696+0.894 \mathrm{RHL}$ & 0.075 & \pm 5.684 & $S=137.388+0.899 \mathrm{RHL}$ & 0.061 & \pm 5.266 \\
\hline & $\operatorname{Men}(n=1,180)$ & $S=138.402+3.239 \mathrm{RHB}$ & 0.101 & \pm 5.605 & $S=128.860+3.196 \mathrm{RHB}$ & 0.082 & \pm 5.207 \\
\hline & Women $(n=1,158)$ & $S=99.632+2.695 \mathrm{RFL}$ & 0.303 & \pm 4.933 & $S=91.659+2.752 \mathrm{RFL}$ & 0.317 & \pm 4.491 \\
\hline & & $S=138.595+2.766 \mathrm{RFB}$ & 0.096 & \pm 5.620 & $S=131.739+2.430 \mathrm{RFB}$ & 0.093 & \pm 5.175 \\
\hline & Northern Han total & $S=151.297+0.896 \mathrm{RHL}$ & 0.069 & \pm 6.035 & $S=143.180+0.758 \mathrm{RHL}$ & 0.043 & \pm 5.738 \\
\hline & Men $(n=5,842)$ & $S=148.537+2.325 \mathrm{RHB}$ & 0.044 & \pm 6.115 & $S=144.388+1.584 \mathrm{RHB}$ & 0.021 & \pm 5.806 \\
\hline & Women $(n=5,892)$ & $S=99.220+2.809 \mathrm{RFL}$ & 0.309 & \pm 5.198 & $S=95.546+2.706 \mathrm{RFL}$ & 0.291 & \pm 4.939 \\
\hline & & $S=146.830+2.186 \mathrm{RFB}$ & 0.060 & \pm 6.065 & $S=137.789+2.113 \mathrm{RFB}$ & 0.055 & \pm 5.704 \\
\hline \multirow{24}{*}{ Southern Han } & $19-29$ & $S=147.299+1.233 \mathrm{RHL}$ & 0.092 & \pm 5.677 & $S=132.785+1.464 \mathrm{RHL}$ & 0.137 & \pm 4.830 \\
\hline & Men $(n=1,595)$ & $S=136.182+4.169 \mathrm{RHB}$ & 0.080 & \pm 5.715 & $S=134.831+3.171 \mathrm{RHB}$ & 0.053 & \pm 5.061 \\
\hline & Women $(n=1,551)$ & $S=86.182+3.439 \mathrm{RFL}$ & 0.439 & \pm 4.462 & $S=88.965+3.098 \mathrm{RFL}$ & 0.372 & \pm 4.120 \\
\hline & & $S=138.041+3.389 \mathrm{RFB}$ & 0.111 & \pm 5.616 & $S=134.683+2.686 \mathrm{RFB}$ & 0.077 & \pm 4.994 \\
\hline & $30-39$ & $S=140.735+1.436 \mathrm{RHL}$ & 0.131 & \pm 5.707 & $S=135.418+1.202 \mathrm{RHL}$ & 0.097 & \pm 4.912 \\
\hline & Men $(n=1,386)$ & $S=132.311+4.191 \mathrm{RHB}$ & 0.086 & \pm 5.855 & $S=125.628+4.093 \mathrm{RHB}$ & 0.092 & \pm 4.925 \\
\hline & Women $(n=1,535)$ & $S=86.462+3.335 \mathrm{RFL}$ & 0.394 & \pm 4.769 & $S=90.586+2.941 \mathrm{RFL}$ & 0.379 & \pm 4.073 \\
\hline & & $S=135.811+3.274 \mathrm{RFB}$ & 0.113 & \pm 5.768 & $S=129.747+2.997 \mathrm{RFB}$ & 0.109 & \pm 4.880 \\
\hline & $40-49$ & $S=133.855+1.740 \mathrm{RHL}$ & 0.218 & \pm 5.541 & $S=130.447+1.452 \mathrm{RHL}$ & 0.136 & \pm 4.997 \\
\hline & Men $(n=1,499)$ & $S=137.005+3.411 \mathrm{RHB}$ & 0.061 & \pm 6.069 & $S=120.344+4.671 \mathrm{RHB}$ & 0.119 & \pm 5.046 \\
\hline & Women $(n=1,613)$ & $S=86.570+3.271 \mathrm{RFL}$ & 0.407 & \pm 4.822 & $S=89.937+2.934 \mathrm{RFL}$ & 0.362 & \pm 4.294 \\
\hline & & $S=129.504+3.748 \mathrm{RFB}$ & 0.148 & \pm 5.783 & $S=131.502+2.704 \mathrm{RFB}$ & 0.084 & \pm 5.143 \\
\hline & $50-59$ & $S=134.437+1.632 \mathrm{RHL}$ & 0.145 & \pm 5.804 & $S=128.132+1.491 \mathrm{RHL}$ & 0.130 & \pm 5.074 \\
\hline & Men $(n=1,442)$ & $S=116.142+5.835 \mathrm{RHB}$ & 0.160 & \pm 5.754 & $S=116.809+4.906 \mathrm{RHB}$ & 0.128 & \pm 5.080 \\
\hline & Women $(n=1,595)$ & $S=87.588+3.186 \mathrm{RFL}$ & 0.386 & \pm 4.920 & $S=85.324+3.071 \mathrm{RFL}$ & 0.396 & \pm 4.227 \\
\hline & & $S=131.340+3.440 \mathrm{RFB}$ & 0.125 & \pm 5.872 & $S=146.830+2.186 \mathrm{RFB}$ & 0.109 & \pm 5.135 \\
\hline & $60+$ & $S=135.000+1.498 \mathrm{RHL}$ & 0.122 & \pm 5.790 & $S=128.641+1.315 \mathrm{RHL}$ & 0.096 & \pm 5.546 \\
\hline & Men $(n=1,457)$ & $S=116.721+5.587 \mathrm{RHB}$ & 0.151 & \pm 5.693 & $S=107.418+5.871 \mathrm{RHB}$ & 0.147 & \pm 5.386 \\
\hline & Women $(n=1,520)$ & $S=87.784+3.119 \mathrm{RFL}$ & 0.362 & \pm 4.934 & $S=78.148+3.309 \mathrm{RFL}$ & 0.384 & \pm 4.578 \\
\hline & & $S=131.838+3.174 \mathrm{RFB}$ & 0.119 & \pm 5.800 & $S=120.112+3.561 \mathrm{RFB}$ & 0.139 & \pm 5.414 \\
\hline & Southern Han total & $S=138.587+1.494 \mathrm{RHL}$ & 0.116 & \pm 6.265 & $S=133.321+1.254 \mathrm{RHL}$ & 0.085 & \pm 5.545 \\
\hline & Men $(n=7,379)$ & $S=134.643+3.792 \mathrm{RHB}$ & 0.062 & \pm 6.456 & $S=134.674+2.702 \mathrm{RHB}$ & 0.037 & \pm 5.687 \\
\hline & Women $(n=7,814)$ & $S=82.335+3.464 \mathrm{RFL}$ & 0.386 & \pm 5.222 & $S=86.651+3.068 \mathrm{RFL}$ & 0.330 & \pm 4.743 \\
\hline & & $S=138.921+2.818 \mathrm{RFB}$ & 0.073 & \pm 6.416 & $S=146.830+2.186 \mathrm{RFB}$ & 0.060 & \pm 5.619 \\
\hline \multirow{4}{*}{ Han Chinese } & Han Chinese total & $S=144.333+1.220 \mathrm{RHL}$ & 0.096 & \pm 6.252 & $S=133.712+2.409 \mathrm{RHL}$ & 0.066 & \pm 5.683 \\
\hline & Men $(n=13,221)$ & $S=140.718+3.147 \mathrm{RHB}$ & 0.057 & \pm 6.385 & $S=139.013+2.196 \mathrm{RHB}$ & 0.031 & \pm 5.788 \\
\hline & Women $(n=13,706)$ & $S=88.522+3.225 \mathrm{RFL}$ & 0.363 & \pm 5.251 & $S=89.811+2.941 \mathrm{RFL}$ & 0.320 & \pm 4.850 \\
\hline & & $S=141.529+2.630 \mathrm{RFB}$ & 0.072 & \pm 6.337 & $S=135.253+2.305 \mathrm{RFB}$ & 0.059 & \pm 5.705 \\
\hline
\end{tabular}

a) $S$, stature; RHL, right hand length; RHB, right hand breadth; RFL, right foot length; RFB, right foot breadth; $R^{2}$, coefficient of determination; SEE, standard error of estimate. 
Table 4 Stepwise regression models and multiple regression models for stature estimation (cm) from right hand/foot measurements in men ${ }^{\text {a) }}$

\begin{tabular}{|c|c|c|c|c|}
\hline \multirow{2}{*}{ Ethnic groups } & \multirow{2}{*}{ Age groups (Years) } & \multicolumn{3}{|l|}{ Men } \\
\hline & & Equation & $R^{2}$ & SEE \\
\hline \multirow{29}{*}{ Northern Han } & $19-29$ & $S=103.689+2.758 \mathrm{RFL}$ & 0.334 & \pm 4.925 \\
\hline & Men $(n=1,131)$ & $S=98.987+2.538 \mathrm{RFL}+0.554 \mathrm{RHL}$ & 0.365 & \pm 4.812 \\
\hline & Women $(n=1,121)$ & $S=135.732+0.91 \mathrm{RHL}+2.341 \mathrm{RHB}$ & 0.149 & \pm 5.572 \\
\hline & & $S=102.477+2.609 \mathrm{RFL}-0.516 \mathrm{RFB}$ & 0.337 & \pm 4.917 \\
\hline & & $S=94.764+2.363 \mathrm{RFL}+0.262 \mathrm{RFB}+0.550 \mathrm{RHL}+0.756 \mathrm{RHB}$ & 0.371 & \pm 4.792 \\
\hline & $30-39$ & $S=101.351+2.761 \mathrm{RFL}$ & 0.345 & \pm 4.587 \\
\hline & Men $(n=1,149)$ & $S=96.084+2.592 \mathrm{RFL}+0.513 \mathrm{RHL}$ & 0.371 & \pm 4.498 \\
\hline & Women $(n=1,193)$ & $S=137.402+0.800 \mathrm{RHL}+1.982 \mathrm{RHB}$ & 0.114 & \pm 5.337 \\
\hline & & $S=102.346+2.880 \mathrm{RFL}-0.410 \mathrm{RFB}$ & 0.347 & \pm 4.582 \\
\hline & & $S=94.174+2.655 \mathrm{RFL}-0.449 \mathrm{RFB}+0.487 \mathrm{RHL}+0.622 \mathrm{RHB}$ & 0.375 & \pm 4.487 \\
\hline & $40-49$ & $S=96.222+2.91 \mathrm{RFL}$ & 0.367 & \pm 4.741 \\
\hline & Men $(n=1,212)$ & $S=90.475+2.724 \mathrm{RFL}+0.563 \mathrm{RHL}$ & 0.395 & \pm 4.636 \\
\hline & Women $(n=1,224)$ & $S=128.804+0.867 \mathrm{RHL}+2.721 \mathrm{RHB}$ & 0.152 & \pm 5.487 \\
\hline & & $S=95.938+2.880 \mathrm{RFL}-0.109 \mathrm{RFB}$ & 0.367 & \pm 4.743 \\
\hline & & $S=88.149+2.618 \mathrm{RFL}+0.045 \mathrm{RFB}+0.555 \mathrm{RHL}+0.558 \mathrm{RHB}$ & 0.397 & \pm 4.630 \\
\hline & $50-59$ & $S=94.392+2.948 \mathrm{RFL}$ & 0.349 & \pm 5.201 \\
\hline & Women $(n=1,196)$ & $S=127.039+0.941 \mathrm{RHL}+2.639 \mathrm{RHB}$ & 0.150 & \pm 5.944 \\
\hline & & $S=93.712+2.884 \mathrm{RFL}-0.232 \mathrm{RFB}$ & 0.350 & \pm 5.199 \\
\hline & & $S=87.181+2.620 \mathrm{RFL}-0.033 \mathrm{RFB}+0.489 \mathrm{RHL}+0.787 \mathrm{RHB}$ & 0.372 & \pm 5.112 \\
\hline & $60+$ & $S=99.632+2.695 \mathrm{RFL}$ & 0.303 & \pm 4.933 \\
\hline & Men $(n=1,180)$ & $S=95.914+2.516 \mathrm{RFL}+0.438 \mathrm{RHL}$ & 0.320 & \pm 4.876 \\
\hline & Women $(n=1,158)$ & $S=127.416+0.756 \mathrm{RHL}+2.885 \mathrm{RHB}$ & 0.153 & \pm 5.441 \\
\hline & & $S=97.740+2.548 \mathrm{RFL}+0.569 \mathrm{RFB}$ & 0.306 & \pm 4.924 \\
\hline & & $S=91.317+2.244 \mathrm{RFL}-0.322 \mathrm{RFB}+0.422 \mathrm{RHL}+1.020 \mathrm{RHB}$ & 0.331 & \pm 4.841 \\
\hline & Northern Han total & $S=99.220+2.809 \mathrm{RFL}$ & 0.309 & \pm 5.198 \\
\hline & Men $(n=5,842)$ & $S=94.988+2.643 \mathrm{RFL}+0.453 \mathrm{RHL}$ & 0.326 & \pm 5.136 \\
\hline & Women $(n=5,892)$ & $S=136.892+0.809 \mathrm{RHL}+1.942 \mathrm{RHB}$ & 0.099 & \pm 5.937 \\
\hline & & $S=100.146+2.899 \mathrm{RFL}-0.327 \mathrm{RFB}$ & 0.310 & \pm 5.194 \\
\hline & & $S=94.650+2.709 \mathrm{RFL}-0.422 \mathrm{RFB}+0.447 \mathrm{RHL}+0.349 \mathrm{RHB}$ & 0.328 & \pm 5.129 \\
\hline \multirow{28}{*}{ Southern Han } & $19-29$ & $S=86.182+3.439 \mathrm{RFL}$ & 0.439 & \pm 4.462 \\
\hline & Men $(n=1,595)$ & $S=83.585+3.286 \mathrm{RFL}+0.350 \mathrm{RHL}$ & 0.446 & \pm 4.437 \\
\hline & Women $(n=1,551)$ & $S=123.070+1.051 \mathrm{RHL}+3.437 \mathrm{RHB}$ & 0.144 & \pm 5.513 \\
\hline & 30-39 & $S=86.462+3.335 \mathrm{RFL}$ & 0.394 & \pm 4.769 \\
\hline & Men $(n=1,386)$ & $S=82.187+3.033 \mathrm{RFL}+0.642 \mathrm{RHL}$ & 0.417 & \pm 4.679 \\
\hline & Women $(n=1,535)$ & $S=118.045+1.241 \mathrm{RHL}+3.208 \mathrm{RHB}$ & 0.179 & \pm 5.550 \\
\hline & & $S=85.744+3.265 \mathrm{RFL}+0.258 \mathrm{RFB}$ & 0.394 & \pm 4.768 \\
\hline & & $S=79.403+2.949 \mathrm{RFL}+0.017 \mathrm{RFB}+0.626 \mathrm{RHL}+0.604 \mathrm{RHB}$ & 0.418 & \pm 4.676 \\
\hline & $40-49$ & $S=86.570+3.271 \mathrm{RFL}$ & 0.407 & \pm 4.822 \\
\hline & Men $(n=1,499)$ & $S=82.121+2.756 \mathrm{RFL}+0.937 \mathrm{RHL}$ & 0.460 & \pm 4.603 \\
\hline & Women $(n=1,613)$ & $S=109.470+1.694 \mathrm{RHL}+3.063 \mathrm{RHB}$ & 0.267 & \pm 5.365 \\
\hline & & $S=85.340+3.133 \mathrm{RFL}+0.478 \mathrm{RFB}$ & 0.409 & \pm 4.817 \\
\hline & & $S=78.441+2.600 \mathrm{RFL}+0.245 \mathrm{RFB}+0.951 \mathrm{RHL}+0.588 \mathrm{RHB}$ & 0.463 & \pm 4.595 \\
\hline & $50-59$ & $S=87.588+3.186 \mathrm{RFL}$ & 0.386 & \pm 4.920 \\
\hline & Men $(n=1,442)$ & $S=83.218+2.864 \mathrm{RFL}+0.666 \mathrm{RHL}$ & 0.406 & \pm 4.839 \\
\hline & Women $(n=1,595)$ & $S=102.426+1.269 \mathrm{RHL}+4.695 \mathrm{RHB}$ & 0.242 & \pm 5.469 \\
\hline & & $S=85.810+3.039 \mathrm{RFL}+0.557 \mathrm{RFB}$ & 0.388 & \pm 4.911 \\
\hline & & $S=74.883+2.536 \mathrm{RFL}+0.214 \mathrm{RFB}+0.618 \mathrm{RHL}+1.829 \mathrm{RHB}$ & 0.420 & \pm 4.786 \\
\hline & $60+$ & $S=87.784+3.19 \mathrm{RFL}$ & 0.362 & \pm 4.934 \\
\hline & Men $(n=1,457)$ & $S=84.435+2.857 \mathrm{RFL}+0.532 \mathrm{RHL}$ & 0.375 & \pm 4.886 \\
\hline & Women $(n=1,520)$ & $S=105.594+1.105 \mathrm{RHL}+4.502 \mathrm{RHB}$ & 0.211 & \pm 5.489 \\
\hline & & $S=86.289+2.969 \mathrm{RFL}+0.532 \mathrm{RFB}$ & 0.365 & \pm 4.926 \\
\hline & & $S=75.683+2.574 \mathrm{RFL}+0.023 \mathrm{RFB}+0.443 \mathrm{RHL}+2.080 \mathrm{RHB}$ & 0.392 & \pm 4.823 \\
\hline & Southern Han total & $S=82.335+3.464 \mathrm{RFL}$ & 0.386 & \pm 5.222 \\
\hline & Men $(n=7,379)$ & $S=78.835+3.202 \mathrm{RFL}+0.542 \mathrm{RHL}$ & 0.399 & \pm 5.166 \\
\hline & Women $(n=7,814)$ & $S=117.913+1.333 \mathrm{RHL}+2.895 \mathrm{RHB}$ & 0.151 & \pm 6.141 \\
\hline & & $S=83.648+3.570 \mathrm{RFL}+0.409 \mathrm{RFB}$ & 0.387 & \pm 5.217 \\
\hline & & $S=78.337+3.286 \mathrm{RFL}-0.567 \mathrm{RFB}+0.539 \mathrm{RHL}+0.480 \mathrm{RHB}$ & 0.401 & \pm 5.158 \\
\hline \multirow{5}{*}{ Han Chinese } & Han Chinese total & $S=88.522+3.225 \mathrm{RFL}$ & 0.363 & \pm 5.251 \\
\hline & Men $(n=13,221)$ & $S=84.618+3.000 \mathrm{RFL}+0.514 \mathrm{RHL}$ & 0.378 & \pm 5.188 \\
\hline & Women $(n=13,706)$ & $S=126.137+1.089 \mathrm{RHL}+2.513 \mathrm{RHB}$ & 0.132 & \pm 6.128 \\
\hline & & $S=89.630+3.323 \mathrm{RFL}-0.368 \mathrm{RFB}$ & 0.364 & \pm 5.247 \\
\hline & & $S=84.128+3.073 \mathrm{RFL}-0.500 \mathrm{RFB}+0.508 \mathrm{RHL}+0.439 \mathrm{RHB}$ & 0.380 & \pm 5.179 \\
\hline
\end{tabular}

a) $S$, stature; RHL, right hand length; RHB, right hand breadth; RFL, right foot length; RFB, right foot breadth; $R^{2}$, coefficient of determination; SEE, standard error of estimate. 
Table 5 Stepwise regression models and multiple regression models for stature estimation (cm) from right hand/foot measurements in women ${ }^{\text {a) }}$

\begin{tabular}{|c|c|c|c|c|}
\hline \multirow{2}{*}{ Ethnic groups } & \multirow{2}{*}{ Age groups (Years) } & \multicolumn{3}{|l|}{ Women } \\
\hline & & Equation & $R^{2}$ & SEE \\
\hline \multirow{30}{*}{ Northern Han } & $19-29$ & $S=98.195+2.703 \mathrm{RFL}$ & 0.338 & \pm 4.443 \\
\hline & Men $(n=1,131)$ & $S=93.009+2.620 \mathrm{RFL}+0.416 \mathrm{RHL}$ & 0.353 & \pm 4.394 \\
\hline & Women $(n=1,121)$ & $S=137.010+0.612 \mathrm{RHL}+1.570 \mathrm{RHB}$ & 0.066 & \pm 5.281 \\
\hline & & $S=99.390+2.818 \mathrm{RFL}-0.445 \mathrm{RFB}$ & 0.340 & \pm 4.438 \\
\hline & & $S=94.512+2.736 \mathrm{RFL}-0.367 \mathrm{RFB}+0.416 \mathrm{RHL}-0.136 \mathrm{RHB}$ & 0.355 & \pm 4.391 \\
\hline & $30-39$ & $S=101.452+2.491 \mathrm{RFL}$ & 0.270 & \pm 4.715 \\
\hline & Men $(n=1,149)$ & $S=96.121+2.380 \mathrm{RFL}+0.459 \mathrm{RHL}$ & 0.291 & \pm 4.651 \\
\hline & Women $(n=1,193)$ & $S=133.799+0.674 \mathrm{RHL}+1.613 \mathrm{RHB}$ & 0.073 & \pm 5.315 \\
\hline & & $S=100.723+2.430 \mathrm{RFL}+0.240 \mathrm{RFB}$ & 0.271 & \pm 4.715 \\
\hline & & $S=94.512+2.736 \mathrm{RFL}-0.367 \mathrm{RFB}+0.416 \mathrm{RHL}-0.136 \mathrm{RHB}$ & 0.291 & \pm 4.652 \\
\hline & $40-49$ & $S=90.904+2.926 \mathrm{RFL}$ & 0.353 & \pm 4.650 \\
\hline & Men $(n=1,212)$ & $S=85.647+2.730 \mathrm{RFL}+0.564 \mathrm{RHL}$ & 0.375 & \pm 4.574 \\
\hline & Women $(n=1,224)$ & $S=121.777+0.996 \mathrm{RHL}+2.381 \mathrm{RHB}$ & 0.133 & \pm 5.385 \\
\hline & & $S=90.241+2.850 \mathrm{RFL}+0.271 \mathrm{RFB}$ & 0.354 & \pm 4.650 \\
\hline & & $S=85.445+2.677 \mathrm{RF}+0.286 \mathrm{RFB}+0.564 \mathrm{RHL}-0.150 \mathrm{RHB}$ & 0.376 & \pm 4.575 \\
\hline & $50-59$ & $S=99.961+2.464 \mathrm{RFL}$ & 0.301 & \pm 4.609 \\
\hline & Men $(n=1,170)$ & $S=96.018+2.308 \mathrm{RFL}+0.433 \mathrm{RHL}$ & 0.316 & \pm 4.561 \\
\hline & Women $(n=1,196)$ & $S=124.532+0.848 \mathrm{RHL}+2.146 \mathrm{RHB}$ & 0.114 & \pm 5.190 \\
\hline & & $S=99.463+2.423 \mathrm{RFL}+0.161 \mathrm{RFB}$ & 0.301 & \pm 4.610 \\
\hline & & $S=94.683+2.239 \mathrm{RFL}+0.100 \mathrm{RFB}+0.434 \mathrm{RHL}+0.262 \mathrm{RHB}$ & 0.316 & \pm 4.563 \\
\hline & $60+$ & $S=91.659+2.752 \mathrm{RFL}$ & 0.317 & \pm 4.491 \\
\hline & Men $(n=1,180)$ & $S=86.751+2.613 \mathrm{RFL}+0.462 \mathrm{RHL}$ & 0.332 & \pm 4.443 \\
\hline & Women $(n=1,158)$ & $S=117.412+0.786 \mathrm{RHL}+2.910 \mathrm{RHB}$ & 0.127 & \pm 5.078 \\
\hline & & $S=90.895+2.648 \mathrm{RFL}+0.353 \mathrm{RFB}$ & 0.318 & \pm 4.488 \\
\hline & & $S=84.675+2.440 \mathrm{RFL}+0.317 \mathrm{RFB}+0.463 \mathrm{RHL}+0.418 \mathrm{RHB}$ & 0.335 & \pm 4.437 \\
\hline & Northern Han total & $S=95.546+2.706 \mathrm{RFL}$ & 0.291 & \pm 4.939 \\
\hline & Men $(n=5,842)$ & $S=91.721+2.602 \mathrm{RFL}+0.360 \mathrm{RHL}$ & 0.301 & \pm 4.907 \\
\hline & Women $(n=5,892)$ & $S=134.349+0.703 \mathrm{RHL}+1.314 \mathrm{RHB}$ & 0.057 & \pm 5.697 \\
\hline & & $S=96.368+2.788 \mathrm{RFL}-0.306 \mathrm{RFB}$ & 0.292 & \pm 4.936 \\
\hline & & $S=94.474+2.744 \mathrm{RFL}-0.122 \mathrm{RFB}+0.372 \mathrm{RHL}-0.685 \mathrm{RHB}$ & 0.305 & \pm 4.894 \\
\hline \multirow{30}{*}{ Southern Han } & $19-29$ & $S=88.965+3.098 \mathrm{RFL}$ & 0.372 & \pm 4.120 \\
\hline & Men $(n=1,595)$ & $S=84.968+2.785 \mathrm{RFL}+0.651 \mathrm{RHL}$ & 0.396 & \pm 4.044 \\
\hline & Women $(n=1,551)$ & $S=120.332+1.324 \mathrm{RHL}+2.088 \mathrm{RHB}$ & 0.158 & \pm 4.771 \\
\hline & & $S=88.968+3.099 \mathrm{RFL}-0.001 \mathrm{RFB}$ & 0.372 & \pm 4.121 \\
\hline & & $S=84.369+2.767 \mathrm{RFL}-0.020 \mathrm{RFB}+0.647 \mathrm{RHL}+0.128 \mathrm{RHB}$ & 0.396 & \pm 4.046 \\
\hline & $30-39$ & $S=90.586+2.941 \mathrm{RFL}$ & 0.379 & \pm 4.073 \\
\hline & Men $(n=1,386)$ & $S=87.933+2.768 \mathrm{RFL}+0.385 \mathrm{RHL}$ & 0.388 & \pm 4.046 \\
\hline & Women $(n=1,535)$ & $S=116.464+0.947 \mathrm{RHL}+3.164 \mathrm{RHB}$ & 0.147 & \pm 4.774 \\
\hline & & $S=89.953+2.881 \mathrm{RFL}-0.227 \mathrm{RFB}$ & 0.379 & \pm 4.073 \\
\hline & & $S=85.809+2.672 \mathrm{RFL}+0.132 \mathrm{RFB}+0.366 \mathrm{RHL}+0.466 \mathrm{RHB}$ & 0.389 & \pm 4.044 \\
\hline & $40-49$ & $S=89.937+2.934 \mathrm{RFL}$ & 0.362 & \pm 4.294 \\
\hline & Men $(n=1,499)$ & $S=85.899+2.632 \mathrm{RFL}+0.631 \mathrm{RHL}$ & 0.384 & \pm 4.221 \\
\hline & Women $(n=1,613)$ & $S=108.607+1.169 \mathrm{RHL}+3.576 \mathrm{RHB}$ & 0.200 & \pm 4.808 \\
\hline & & $S=89.204+2.884 \mathrm{RFL}+0.21 \mathrm{RFB}$ & 0.362 & \pm 4.294 \\
\hline & & $S=79.143+2.483 \mathrm{RFL}-0.181 \mathrm{RFB}+0.554 \mathrm{RHL}+1.739 \mathrm{RHB}$ & 0.396 & \pm 4.179 \\
\hline & $50-59$ & $S=85.324+3.071 \mathrm{RFL}$ & 0.396 & \pm 4.227 \\
\hline & Men $(n=1,442)$ & $S=82.039+2.823 \mathrm{RFL}+0.516 \mathrm{RHL}$ & 0.409 & \pm 4.182 \\
\hline & Women $(n=1,595)$ & $S=106.765+1.128 \mathrm{RHL}+3.683 \mathrm{RHB}$ & 0.194 & \pm 4.885 \\
\hline & & $S=83.990+2.969 \mathrm{RFL}+0.412 \mathrm{RFB}$ & 0.398 & \pm 4.223 \\
\hline & & $S=76.872+2.641 \mathrm{RFL}+0.131 \mathrm{RFB}+0.445 \mathrm{RHL}+1.241 \mathrm{RHB}$ & 0.416 & \pm 4.159 \\
\hline & $60+$ & $S=78.148+3.309 \mathrm{RFL}$ & 0.384 & \pm 4.578 \\
\hline & Men $(n=1,457)$ & $S=76.152+3.147 \mathrm{RFL}+0.327 \mathrm{RHL}$ & 0.389 & \pm 4.561 \\
\hline & Women $(n=1,520)$ & $S=99.660+0.893 \mathrm{RHL}+4.864 \mathrm{RHB}$ & 0.187 & \pm 5.260 \\
\hline & & $S=75.470+3.057 \mathrm{RFL}+0.947 \mathrm{RFB}$ & 0.392 & \pm 4.552 \\
\hline & & $S=67.460+2.751 \mathrm{RFL}+0.639 \mathrm{RFB}+0.238 \mathrm{RHL}+1.799 \mathrm{RHB}$ & 0.407 & \pm 4.498 \\
\hline & Southern Han total & $S=86.651+3.068 \mathrm{RFL}$ & 0.330 & \pm 4.743 \\
\hline & Men $(n=7,379)$ & $S=84.412+2.900 \mathrm{RFL}+0.351 \mathrm{RHL}$ & 0.336 & \pm 4.723 \\
\hline & Women $(n=7,814)$ & $S=123.789+1.105 \mathrm{RHL}+1.636 \mathrm{RHB}$ & 0.097 & \pm 5.507 \\
\hline & & $S=87.598+3.148 \mathrm{RFL}-0.315 \mathrm{RFB}$ & 0.331 & \pm 4.740 \\
\hline & & $S=87.130+3.014 \mathrm{RFL}-0.181 \mathrm{RFB}+0.383 \mathrm{RHL}-0.573 \mathrm{RHB}$ & 0.338 & \pm 4.716 \\
\hline \multirow{5}{*}{ Han Chinese } & Han Chinese total & $S=89.811+2.941 \mathrm{RFL}$ & 0.320 & \pm 4.850 \\
\hline & Men $(n=13,221)$ & $S=86.733+2.794 \mathrm{RFL}+0.373 \mathrm{RHL}$ & 0.328 & \pm 4.822 \\
\hline & Women $(n=13,706)$ & $S=127.792+0.919 \mathrm{RHL}+1.597 \mathrm{RHB}$ & 0.081 & \pm 5.636 \\
\hline & & $S=90.782+3.028 \mathrm{RFL}-0.335 \mathrm{RFB}$ & 0.321 & \pm 4.846 \\
\hline & & $S=89.455+2.928 \mathrm{RFL}-0.183 \mathrm{RFB}+0.394 \mathrm{RHL}-0.603 \mathrm{RHB}$ & 0.330 & \pm 4.812 \\
\hline
\end{tabular}

a) $S$, stature; RHL, right hand length; RHB, right hand breadth; RFL, right foot length; RFB, right foot breadth; $R^{2}$, coefficient of determination; SEE, standard error of estimate. 
were lower than those of linear regression equations. The finding is in agreement with the results of previous studies. The multiple regression equations of the northern Han people were as follows: $S=94.650+2.709 \mathrm{RFL}-0.422 \mathrm{RFB}+$ $0.447 \mathrm{RHL}+0.349 \mathrm{RHB}$ for men and $S=94.474+2.744 \mathrm{RFL}$ $-0.122 \mathrm{RFB}+0.372 \mathrm{RHL}-0.685 \mathrm{RHB}$ for women. The multiple regression equations of the southern Han people were as follows: $S=78.337+3.286 \mathrm{RFL}-0.567 \mathrm{RFB}+0.539 \mathrm{RHL}+$ $0.480 \mathrm{RHB}$ for men and $S=87.130+3.014 \mathrm{RFL}-0.181 \mathrm{RFB}$ $+0.383 \mathrm{RHL}-0.573 \mathrm{RHB}$ for women.

Estimation of stature from right hand and right foot dimensions by using regression equations has great implications and usefulness for physical and forensic anthropology. Because there is no sampling study for left hand/foot dimensions, the right foot lengths would provide the most reliable stature estimation. This study provides new forensic standards for the estimation of stature from the right hand and right foot measurements of Han Chinese adults.

\section{MATERIALS AND METHODS}

\section{Materials}

In this study, measurements taken from 26,927 healthy adult subjects (13,221 men, 13,706 women) currently residing in China were examined. The subjects were required to sign a consent form and complete a questionnaire containing basic demographic data and general questions, e.g., sex, age, and ethnicity. The subjects were between 19 and 75 years of age (mean age of men: $45.1 \pm 16.3$ years, mean age of women: $45.0 \pm 15.6$ years). All subjects were healthy local Han Chinese people who were native to their areas of residence for more than three generations. The modern Chinese population can be divided into the northern and southern groups, separated by Qinling Mountains and Huaihe River. The study included 11,734 northern Han (5,842 men, 5,892 women) and 15,193 southern Han (7,379 men, 7,814 women) subjects. The survey samples were chosen by means of random cluster sampling. These Han Chinese subjects were categorized in five subgroups according to their age ranges: 19-29, 30-39, $40-49,50-59$, and 60 years and older. The sampling locations included Sichuan, Inner Mongolia, Guangdong, Liaoning, Henan, Hebei, Shaanxi, Gansu, Jiangxi, Jiangsu, Hunan, Hainan, Anhui, Shanxi, Shandong, Zhejiang, Fujian, Hubei, Yunnan, Guizhou, Jilin, and Heilongjiang.

\section{Methods}

\section{Measurements}

Stature was measured by using an anthropometer. For stature, the distance from the vertex to the floor in the anatomical position with the head oriented in the Frankfurt plane was measured (Figure 1) (Martin and Saller, 1957).

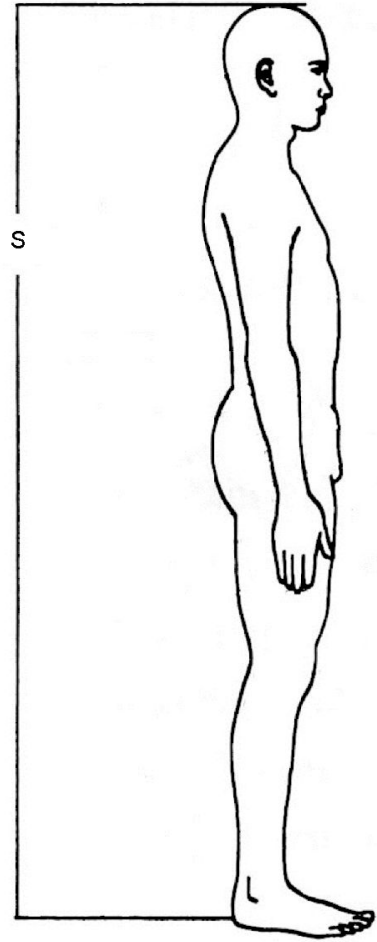

Figure 1 Stature (S): the distance from the vertex to the floor in the anatomical position with the head oriented in the Frankfurt plane.

For hand length, the distance between the midpoint of the distal transverse crease of the wrist to the most anterior projection of the skin of the middle finger was measured (Ishak et al., 2012).

For hand breadth, the distance between the most lateral point on the head of the second metacarpal to the most medial point on the head of the fifth metacarpal was measured (Ishak et al., 2012).

For foot length, the maximum distance between the heel (pternion) and the longest toe (akropodian) (Hemy et al., 2013) was measured.

For foot breadth, the distance between the most prominent point on the medial side of the foot to the most prominent point on the lateral side (which corresponds to the heads of the first to fifth metatarsals) (Hemy et al., 2013) was measured.

With respect to establishing the reliability of obtaining stature and right hand and right foot measurements, we have verified that the degree of measurement error and reliability are well within acceptable standards $(R>0.9 ;$ rTEM $<5 \%)$ (Perini et al., 2005; Knapp, 1992; Reynolds et al., 2008).

\section{Statistical analysis}

The data were statistically analyzed by using SPSS 17.0 for Windows and Microsoft Office Excel 2003. The significance of sex differences was tested by using an independent $t$-test. The association between stature and right hand or right foot measurements was determined by using Pearson correlation 
analysis. Linear regression analysis, stepwise regression, and multiple regression analyses were used to calculate the equations for the estimation of stature from right hand and right foot dimensions.

Compliance and ethics The author(s) declare that they have no conflict of interest.

Acknowledgements We would like to thank all the volunteers who participated in this project. This work was supported by the National Natural Science Foundation of China (30830062, 31401022).

Abdel-Malek, A.K., Ahmed, A.M., El Aziz El Sharkawi, S.A., and El Maksoud Abd El Hamid, N.A. (1990). Prediction of stature from hand measurements. Forensic Sci Int 46, 181-187.

Agnihotri, A.K., Agnihotri, S., Jeebun, N., and Googoolye, K. (2008). Prediction of stature using hand dimensions. J Forensic Legal Med 15, 479-482.

Agnihotri, A.K., Purwar, B., Googoolye, K., Agnihotri, S., and Jeebun, N. (2007). Estimation of stature by foot length. J Forensic Legal Med 14, 279-283.

Ahmed, A.A. (2013). Estimation of stature from the upper limb measurements of Sudanese adults. Forensic Sci Int 228, 178.e1-178.e7.

Ahmed, A.A. (2013). Estimation of stature using lower limb measurements in Sudanese Arabs. J Forensic Legal Med 20, 483-488.

Aldegheri, R., and Dall'Oca,C. (2001). Limb lengthening in short stature patients. J Pediatr Orthop B 10, 238-247.

Atamturk, D., and Duyar, I. (2008). Age-related factors in the relationship between foot measurements and living stature and body weight. J Forensic Sci 53, 1296.

Baume, R. M., and Buschang, P.H. (1983). Weinstein S. Stature, head height, and growth of the vertical face. Am J Orthod 83, 477-484.

Cervantes, C.D., Lifshitz, F., and Levenbrown, J. (1988). Radiologic anthropometry of the hand in patients with familial short stature. Pediatr Radiol 18, 210-214.

Choi, B.Y., Chae, Y.M., Chung, I.H., and Kang, H.S. (1997). Correlation between the postmortem stature and the dried limb-bone lengths of Korean adult males. Yonsei Med J 38, 79.

Fawzy, I.A., and Kamal, N.N. (2010). Stature and body weight estimation from various footprint measurements among egyptian population. J Forensic Sci 55, 884-888.

Fessler, D.M.T., Haley, K.J., and Lal, R.D. (2005). Sexual dimorphism in foot length proportionate to stature. Ann Human Biol 32, 44-59.

Gordon, C.C., and Buikstra, J.E. (1992). Linear models for the prediction of stature from foot and boot dimensions. J Forensic Sci 37, 771-782.

Habib, S.R., and Kamal, N.N. (2010). Stature estimation from hand and phalanges lengths of Egyptians. J Forensic Legal Med 17, 156-160.

Hautvast, J. (1971). Growth in stature and head and face measurements in Dutch children aged 7 to 14 . Hum Biol 43, 340-343.

Hemy, N., Flavel, A., Ishak, N.I., and Franklin, D. (2013). Sex estimation using anthropometry of feet and footprints in a Western Australian population. Forensic Sci Int 231, 402.e1-402.e6.

Ikeda, Y., Higurashi, M., Hirayama, M., Ishikawa, N., and Hoshina, H. (1977). A longitudinal study on the growth of stature, lower limb and upper limb length in Japanese children with Down's syndrome. J Ment Defic Res 21, 139-151.

Ishak, N.I., Hemy, N., and Franklin, D. (2012). Estimation of stature from hand and handprint dimensions in a Western Australian population. Forensic Sci Int 216, 199.e1-199.e7.

Ishak, N.I., Hemy, N., and Franklin, D. (2012). Estimation of sex from hand and handprint dimensions in a Western Australian population. Forensic Sci Int 221, 154.e1-154.e6.
Jason, D.R., and Taylor, K. (1995). Estimation of stature from the length of the cervical, thoracic, and lumbar segments of the spine in American whites and blacks. J Forensic Sci 40, 59-62.

Jasuja, O.P., Singh, J., and Jain, M. (1991). Estimation of stature from foot and shoe measurements by multiplication factors: a revised attempt. Forensic Sci Int 50, 203-215.

Kanchan, T., Krishan, K., Sharma, A., and Menezes, R.G. (2010). A study of correlation of hand and foot dimensions for personal identification in mass disasters. Forensic Sci Int 199, 112.e1-112.e6.

Kanchan, T., Menezes, R.G., Lobo, S.W., and Kotian, M.S. (2010). Forensic anthropology population data: stature estimation from foot measurements - Comparison of error in sex dependent and independent models. Forensic Sci Int 194, e29.

Kanchan, T., Menezes, R.G., Moudgil, R., Kaur, R., Kotian, M.S., and Garg, R.K. (2008). Stature estimation from foot dimensions. Forensic Sci Int 179, 241.e1-241.e5.

Kanchan, T., Menezes, R.G., Moudgil, R., Kaur, R., Kotian, M.S., and Garg, R.K. (2010). Stature estimation from foot length using universal regression formula in a North Indian population. J Forensic Sci 55, 163-166.

Karakas, H.M., Celbis, O., Harma, A., and Alicioglu, B. (2011). Total body height estimation using sacrum height in Anatolian Caucasians: multidetector computed tomography-based virtual anthropometry. Skeletal Radiol 40, 623-630.

Knapp, T.R. (1992). Technical error of measurement: a methodological critique. Am J Phys Anthropol 87, 235-236.

Krishan, K. (2008a). Determination of stature from foot and its segments in a North Indian population. Am J Forensic Med Pathol 29, 297-303.

Krishan, K. (2008b). Estimation of stature from footprint and foot outline dimensions in Gujjars of North India. Forensic Sci Int 175, 93-101.

Krishan, K., Kanchan, T., and Sharma, A. (2012). Multiplication factor versus regression analysis in stature estimation from hand and foot dimensions. J Forensic Legal Med 19, 211-214.

Krishan, K., and Sharma, A. (2007). Estimation of stature from dimensions of hands and feet in a North Indian population. J Forensic Legal Med 14, 327-332.

Lavelle, C.I. (1977). Stature, limb and jaw growth. Acta Anatomica 98, 97-100.

Mahakkanukrauh, P., Khanpetch, P., Prasitwattanseree, S., Vichairat, K., and Troy Case, D. (2011). Stature estimation from long bone lengths in a Thai population. Forensic Sci Int 210, 279.e1-279.e7.

Martin, R., and Saller, K. (1957). Lehrbuch der Anthropologie. Stuttgart: Gustav Fischer Verlag.

Moorthy, T.N., Mostapa, A.M.B., Boominathan, R., and Raman, N. (2014). Stature estimation from footprint measurements in Indian Tamils by regression analysis. Egyptian J Forensic Sci 4, 7-16.

Nagesh, K.R., and Pradeep Kumar, G. (2006). Estimation of stature from vertebral column length in South Indians. Legal Med 8, 269-272.

Ozden, H., Balci, Y., Demirüstü, C., Turgut, A., and Ertugrul, M. (2005). Stature and sex estimate using foot and shoe dimensions. Forensic Sci Int 147, 181-184.

Pelin, C., Duyar, I., Kayahan, E.M., Zagyapan, R., Agildere, A.M., and Erar, A. (2005). Body height estimation based on dimensions of sacral and coccygeal vertebrae. J Forensic Sci 50, 294-297.

Perini, T.A., Oliveira, G.L., Ornellas, J.S., and Oliveira, F.P. (2005). Cálculo do erro técnico de medição em antropometria. Rev Bras Med Esporte 11, $81-85$.

Pininski, M., and Brits, D. (2014). Estimating stature in South African populations using various measures of the sacrum. Forensic Sci Int 234, 182.e1-182.e7.

Qing, S., Chang, Y., Dong, X., Li, Y., Chen, X., Shu, Y., and Deng, Z. (2013). Stature estimation for Sichuan Han nationality female based on X-ray technology with masurement of lumbar vertebrae. Fa Yi Xue Za Zhi 29, 344-347.

Rastogi, P., Nagesh, K.R., and Yoganarasimha, K. (2008). Estimation of stature from hand dimensions of north and south Indians. Legal Med 10, 
185-189.

Reel, S., Rouse, S., Vernon OBE, W., and Doherty, P. (2012). Estimation of stature from static and dynamic footprints. Forensic Sci Int 219, 283.e1-283.e5.

Reynolds, M., Franklin, D., Raymond, M.A., and Dadour, I. (2008). Bloodstain measurement using computer fitted theoretical ellipses a study in accuracy and precision. J Forensic Ident 58, 469-484.

Sahni, D., Sanjeev, D., Sharma, P., Harjeet, P., Kaur, G., and Aggarwal, A. (2010). Estimation of stature from facial measurements in northwest Indians. Legal Med 12, 23-27.

Sanli, S.G., Kizilkanat, E.D., Boyan, N., Ozsahin, E.T., Bozkir, M.G., Soames, R., Erol, H., and Oguz, O. (2005). Stature estimation based on hand length and foot length. Clin Anat 18, 589-596.

Saxena, S.K. (1984). A study of correlations and estimation of stature from hand length, hand breadth and sole length. Anthropol Anz 42, 271-276.

Sen, J., and Ghosh, S. (2008). Estimation of stature from foot length and foot breadth among the Rajbanshi: an indigenous population of North Bengal. Forensic Sci Int 181, 55.e1-55.e6.

Stanitski, C.L. (2004). Limb lengthening for stature. J Pediatric Orthopaedics 24, 593-594.

Terazawa, K., Akabane, H., Gotouda, H., Mizukami, K., Nagao, M., and Takatori, T. (1990). Estimating stature from the length of the lumbar part of the spine in Japanese. Med Sci Law 30, 354-357.

Tibbetts, G. L. (1981). Estimation of stature from the vertebral column in American Blacks. J Forensic Sci 26, 715-723.

Torimitsu, S., Makino, Y., Saitoh, H., Ishii, N., Hayakawa, M., Yajima, D., Inokuchi, G., Motomura, A., Chiba, F., and Iwase, H. (2014). Stature estimation in Japanese cadavers using the sacral and coccygeal length measured with multidetector computed tomography. Legal Med 16, 14-19.
Trotter, M., and Gleser, G.C. (1958). A re-evaluation of estimation of stature based on measurements of stature taken during life and of long bones after death. Am J Phys Anthropol 16, 79-123.

Uhrová, P., Beňuš, R., Masnicová, S., Obertová, Z., Kramárová, D., Kyselicová, K., Dörnhöferová, M., Bodoriková, S., and Neščáková, E. (2015). Estimation of stature using hand and foot dimensions in Slovak adults. Legal Med 17, 92-97.

Uhrová, P., Beňuš, R., and Masnicová, S. (2013). Stature estimation from various foot dimensions among slovak population. J Forensic Sci 58, $448-451$.

Zaher, J.F., El-Ameen, N.F.M., and Seedhom, A.E. (2011). Stature estimation using anthropometric measurements from computed tomography of metacarpal bones among Egyptian population. Egyptian J Forensic Sci 1, 103-108.

Zeybek, G., Ergur, I., and Demiroglu, Z. (2008). Stature and gender estimation using foot measurements. Forensic Sci Int 181, 54.e1-54.e5.

Zhang, K., Chang, Y., Fan, F., and Deng, Z. (2015). Estimation of stature from radiologic anthropometry of the lumbar vertebral dimensions in Chinese. Legal Med 17, 483-488.

Zhang, Z., Chang, Y., Zhou, X., Deng, Z., Yu, J., and Huang, L. (2008). Huang, Stature estimation from the cervical vertebrae of living male by measuring Xray films of computer radiography. Fa Yi Xue Za Zhi 24, 25-31.

Zheng, L.B., Li, Y.L., Xi, H.J., Yu, K.L., Lu, S.H., Shi, R., Wen, Y.F., Bao, J.P., Zhang, X.H., Li, Y.L., Ren, F., and Xu, G.C. (2015). Statures in Han populations of China. Sci China Life Sci 58, 215-217.

Zheng, L.B., Li, Y.L., Lu, S.H., Bao, J.P., Wang, Y., Zhang, X.R., Xue, H., and Rong, W.G. (2013). Physical characteristics of Chinese Hakka. Sci China Life Sci 56, 541-551. 